\title{
Distribuição geográfica de Leucobryaceae (Bryopsida) na Amazônia
}

\author{
Olga Yano (')
}

\begin{abstract}
Resumo
Dezenove espécies e uma variedade de Leucobryaceae são relatadas para a Amazônia brasileira. Destas, nove espécies constituem a primeira citação para a região: Leucobryum albidum ( $P$. Beauv.) Lindb., L. bowringii Mitt., L. giganteum C. Muell., L. juniperoideum (Brid.) C. Muell., Octoblepharum albidum var. violascens C. Muell., O. africanum (Broth.) Card., O. erectifolium Mitt. ex William, $\mathbf{O}$. rhaphidostegium $\mathrm{C}$. Muell. ex Broth. e Ochrobryum obtusifolium (C. Muell.) Mitt. As espécies que ocorrem com maior freqüência na região estudada são: Leucobryum martianum (Hornsch.) Hampe, Octoblepharum pulvinatum (Dozy et Molk.) Mitt. e $\mathbf{O}$. albidum Hedw.
\end{abstract}

\section{INTRODUÇÃO}

A região amazônica brasileira ou Amazônia Legal, compreende os Estados do Acre, Amazonas, Pará; os Territórios do Amapá, Rondônia e Roraima. Estão também incluidos os Estados do Maranhão no limite $44^{\circ}$ Long. W, Goiás $13^{\circ}$ Lat. $\mathrm{S}$ e Mato Grosso $16^{\circ}$ Lat. S.

Serviram como base para este levantamento, o trabalho de Spruce (1867), com uma listagem de musgos da região amazônica e dos Andes. Em seguida o trabalho de Mitten (1869), musgos para a América do Sul. baseado principalmente nas coletas de Spruce, mencionando as seguintes espécies de Leucobryaceae: Ochrobryum gardneri (C. Muell.) Lindb., Octoblepharum albidum Hedw., O. cocuiense Mitt., $O$. cylindricum Mont., $O$. Iongifolium Mitt., O. pulvinatum (Dozy et Molk.) Mitt. e Leucobryum martianum (Hornsch.) Hampe; além de apresentar uma chave de identificação das espécies.

Ducke \& Black (1954), em estudo sobre a fitogeografia da Amazônia Brasileira, mencionam que, entre as plantas mais notáveis das "campinas", é encontrado Sphagnum sp.
Prance (1971), apresenta uma lista individual de coletores da Amazônia, e o que se observa é que poucos briologistas têm trabaIhado na Amazônia Central desde o tempo de Spruce; enquanto que várias publicações de flora ou taxonomia de fanerógamos têm surgido recentemente.

Só agora, as coletas de briófitas foram intensificadas na região. Assim cita-se o periodo de 1974, com material principalmente da região do Município de Manaus. Os anos de 1977 e 1978 respectivamente, são representados na área pelas coleções de W.R. Buck e W.R. Reese. O restante das exsicatas é proveniente de coletas mais esporádicas realizadas por diversos pesquisadores e mateiros.

Outros trabalhos mais recentes têm apresentado sobre a brioflora da Campina (Lisboa, 1976); uma lista de musgos da Reserva de Aripuanã (Lisboa \& Lisboa, 1978); sobre Calymperaceae para a Amazônia (Reese \& Griffin, 1976 e 1977 e Reese, 1979); e uma chave de identificação das briófitas de Manaus (Griffin, 1979).

Para a identificação das Leucobryaceae da Amazônia, foram utilizados os trabalhos de Bescherelle (1897), Florschütz (1964), Yano (1975) e Griffin (1979).

O objetivo deste trabalho é contribuir para o inventário da brioflora da Amazônia.

As exsicatas dos materiais se encontram nos herbários das seguintes instituições: Instituto Nacional de Pesquisas da Amazônia (INPA), Instituto de Botânica de São Paulo (SP), Museu Paraense Emílio Goeldi (MG), Instituto de Pesquisa e Experimentação Agropecuária do Norte, Belém (IAN), University of Florida (FLAS), The New York Botanical Garden (NY) e University of Southwestern Louisiana (LAF).

(1) - Instituto de Botânica, São Paulo. 


\section{ESPÉCIMES ENCONTRADOS}

Leucobryum albidum (P. Beauv.) Lindb. Mato Grosso: Mun. de Aripuanã, Dardanelos, col. P. Lisboa et al. 279, 26-IX-1975, det. O. Yano (INPA52760, SP147624); Mun. de Aripuanã, Dardanelos, antiga picada do aeroporto, col. P. Lisboa et al. 503, 30-IX-1975, det. R. Lisboa (INPA52908, SP147630); Mun. de Aripuanã, Dardanelos, col. P. Lisboa et al. 547, 1-X-1975, det. R. Lisboa (INPA52666, SP147621); Mun. de Aripuanã, col. C. Damião 762, 14-II-1977, det. R. Lisboa (INPA66207, SP147689); Mun. de Aripuanã, col. C. Damião 771 , 18-Il-1977, det. R. Lisboa (INPA66216, SP147692); Mun. de Aripuanã, col. C. Damião 780, 785, 19-II-1977, det. R. Lisboa (INPA66225, SP147695, INPA66230, SP147698); Mun. de Aripuanä, Est. Humboldt, col. C. Damião 2469, 28-III-1977, det. O. Yano (INPA69495, SP147708); Mun. de Aripuanã, Est. Humboldt, col. C. Damião 2471, 25-III-1977, det. O. Yano (INPA69497, SP147710); Mun. de Aripuanã, Est. Humboldt, col. C. Damião 2476, 26-III-1977, det. R. Lisboa (INPA69502, SP147713); Vicinity of Colégio Buriti, Chapada dos Guimarães, disturbed dry forest, alt. $720 \mathrm{~m}$, col. G. T. Prance et al. 19020, 15-X-1973, det. O. Yano (INPA42511, NY, SP148745); Gorge of Véu de Noiva, Chapada dos Guimarães, col. G.T. Prance et al 19091, 19093, 17-X-1973, det. O. Yano (INPA42582, 42584, NY); Vicinity of Buriti, Chapada dos Guimarães, alt. $720 \mathrm{~m}$, disturbed forest, col. G.T. Prance et al. 19249, 22-X-1979, det. O. Yano (INPA42741, NY); $5 \mathrm{~km}$ $E$ of town of Chapada dos Guimarães on road to EMBRATEL, disturbed forest on south facing slope of Chapada, alt. $720 \mathrm{~m}$, col. G.T. Prance et al. 19376, 24-X-1973, det. O. Yano (INPA42867, NY); Véu de Noiva, Chapada dos Guimarães, alt. $720 \mathrm{~m}$, col. G.T. Prance et al. 19396, 25.X-1973, det. O. Yano (INPA42887, NY). Pará: Planalto de Santarém, localidade Sertão, col. R.L. Fróes 31113, ?-X-1954, det. O. Yano (IAN86369); Serra do Cachimbo, BR-163, Campina de areia branca, $\mathrm{km} 832$, col. E. Lleras \& J. H. Kirkbride 1172, 16-II-1977, det. O. Yano (INPA64326). Território de Rondônia: secondary forest, col. P.J. \& H. Maas 389, 19-VIII-1971, det. O. Yano (INPA34939). L. albidum está sendo mencionada pela primeira vez na região amazônica. Crescem nos paredões da cachoeira, tronco de árvore viva ou morta, sobre rochas e na base das árvores. Reconhece-se facilmente pelo tamanho (1-3cm compr.); os filídios imbricados $4,5 \mathrm{~mm}$ compr. Em corte transversal do filídio possuem 4 camadas de leucocistos na parte mais espessada.

Luecobryum bowringii Mitt. Território de Roraima: Summit of Serra Parima, South of Auaris, $1200 \mathrm{~m}$ alt., cloud forest, col. G.T. Prance et al. 21595, 30-VII-1974, det. O. Yano (INPA45526). Esta espécie é a primeira menção para a região amazônica; foi encontrada crescendo na base de árvore; são plantas pequenas $\pm 1,5 \mathrm{~mm}$. Os filídios patentes e flexuosos; as células da lâmina apresentam constricções de espaço em espaço.

Leucobryum crispum C. Muell. Amazonas: Manaus, ao longo da rodovia Manaus-Caracaraí, km 130, col. D. Griffin, D.M. Vital \& O. Yano 421, 25-VII-1974, det. O. Yano (SP125417). Mato Grosso: $5 \mathrm{~km} \mathrm{E}$ of town of Chapada dos Guimarães on road to EMBRATEL, disturbed forest on south facing slope of Chapeda, alt. $720 \mathrm{~m}$, col. G.T. Prance et al. 19391, 24-X-1973, det. O. Yano (INPA42882, NY). Território de Roraima: Serra da Lua $2^{\circ} 25^{\prime}-29^{\prime} \mathrm{N}, 60^{\circ} 11-14^{\prime} \mathrm{W}$ upper slopes of Serra da Lua $1200 \mathrm{~m}$, col. G.T. Prance et al. 9450pp, 9461, 24-I-1969 (INPA26587, 26598, mixed Rhizogonium spiniforme (Hedw.) Bruch); Serra Parima, col. Bruce W. Nelson 21595, 28.VII-1974, det. O. Yano (SP125544); Paracaima, vicinity of $2 .^{\circ}$ BEF Army Encampment $226 \mathrm{~km} \mathrm{~N}$ of Boa Vista just $\mathrm{S}$ of Venezuela border at Paracaima on Boa VistaCaracas Road, col. W.R. Buck 1945, 28-X!-1977; det. O. Yano (SP147797). A espécie foi citada pela $10^{\circ}$ vez por Lisboa (1976). E relativamente fácil reconhecer por possuir os filídios poucos falcados e pelo corte transversal do filídio na base de 3-4 camadas de leucocistos e nas outras regiões 2 camadas. Crescem em solo humoso, rochas úmidas, barrancos de rios e em troncos de árvores.

Leucobryum giganteum C. Muell. Amazonas: Serra Pirapucu, alt. $1250 \mathrm{~m}$, rio Negro, rio Cauaburi, rio Maturacá, col. Nilo T. Silva \& Umbelino Brazão, 60910, 27-I-1966 (NY, SP148752); Basin of rio Negro, summits of 
Serra Curicuriari, col. G.T. Prance et al. 16094, 5-XI-1971, det. O. Yano (INPA34313). Reconhece-se facilmente pelo tamanho do filídio (até $20 \mathrm{~mm}$ compr.), com as bases ovado-elipticas e o ápice subtubuloso. Crescem em rochas e árvores mortas formando pequenos coxins. $\hat{E}$ a primeira vez citada para a região amazônica.

Leucobryum juniperoideum (Brid.) C. Muell. Acre: Cruzeiro do Sul, próximo ao aeroporto Novo, Projeto Radam/Brasil, col. O.P. Monteiro \& C. Damião 387, 14-II-1976, def. O. Yano teiro \& C. Damião 550, 20-11-1976, det. O. Yano (INPA56237, SP157650); Território de Roraima, Boca da Mata, $216 \mathrm{~km} \mathrm{~N}$ of Boa Vista on Boa Vista-Caracas Road, col. W.R. Buck 1952, 29-XI-1977, det. O. Yano (SP147798). Esta espécie possui a cor verde-azulado mesmo quando seco, mede de $7-9 \mathrm{~mm}$ de altura. Crescem formando pequenos tufos em rochas, árvores viva e em rochas ao longo dos rios. Os filídios possuem 5 fileiras de células hialinas na margem. Está sendo citada pela $1 .^{\circ}$ vez na Amazônia.

Leucobryum martianum (Hornsch.) Hampe. Acre: Cruzeiro do Sul, Estrada Alemanha, col. G.T. Prance et al. 11864, 14-IV-1971, det. O. Yano (INPA30177); Cruzeiro do Sul, Estrada Alemanha, col. G.T. Prance et al. 11928, 29-IV-1971, det. O. Yano (INPA30241); Cruzeiro do Sul, Vicinity of Serra da Moa village, col. G.T. Prance et al. 12608, 29-IV-1971, det. O. Yano (INPA30930); Cruzeiro do Sul, Estrada Alemanha, col. P.J.M. Maas et al. P12792, 8-V-1971, det. O. Yano (INPA31154); Cruzeiro do Sul, próximo ac aeroporto Novo, Projeto Radam/Brasil, col. O.P. Monteiro \& C. Damião 415, 15-II-1976, det. O. Yano (INPA56265, SP147651); Cruzeiro do Sul, próximo novo aeroporto, Projeto Radam/Brasil, col. O.P. Monteiro \& C. Damião 550, 20-II-1976, det. O. Yano (INPA56400, SP147652); Cruzeiro do Sul col. N.A. Rosa 661, 30-II-1976 (IAN151271). Amazonas: Manaus, cachoeira Baixa do Tarumã, 16 $\mathrm{km}$ North of Manaus, col. G.T. Prance et al. 2282, 12-IX-1966 (INPA18765); Manaus, Peserva Florestal Ducke, km 27 Manaus-Itacoatiara Road, col. G.T. Prance et al. 9020, 12-XII-1968, det. O. Yano (INPA25819, SP147599); Manaus, Reserva Florestal Ducke, km 27, Manaus-Ita- coatiara road, col. G.T. Prance et al. 11303, 11313, 11314, 2-IV-1971 (INPA29703, SP147601; NY, SP148754; INPA29714, SP147604); Manaus, Manaus-Caracaraí road, km 48, col. G.T. Prance et al. 11400, 5-IV-1971 (INPA29800); Basin of rio Purus, rio Cunha et Deni Indian village, $6^{\circ} 43^{\prime} \mathrm{S}, 66^{\circ} 47^{\prime} \mathrm{W}$, forest beside stream, col. G.T. Prance et al. 16511, 29-XI-1971, det. O. Yano (INPA34731); Manaus, Ponta Negra, col. G.T. Prance 11699, 11713, 31-III-1971, det. O. Yano (INPA30011, 30025); Ponta Negra, col. G.T. Prance et al. 11702, 31-III-1971, det. O. Yano (INPA30014); Rio Purus between Lago Quati \& Lago Arimã, near rio Jacaré, col. G.T. Prance 13417, 20-VI-1971, det. O. Yano (INPA31781); Fortaleza, rio Puciari, tributary of rio Ituxi, $20 \mathrm{~km}$ above mouth, col. G.T. Prance et al. 13862, 1-VII-1971, (INPA32079); Rio Ituxi, vicinity of Boca do Curuquetê, col. G.T. Prance et al. 14167, 12-VII-1971 (INPA32382); Basin of rio Negro, rio Cuieras, just below mouth of rio Bracinho, savana forest on sandy soil, col. G.T. Prance, D.F. Coelho \& O.P. Monteiro 14802, 24-IX-1971 (INPA33018); Rio Cuieras just below mouth of rio Bracinho, high campina on sandy soil, col. G.T. Prance, D.F. Coelho \& O.P. Monteiro 14804, 24-IX-1971 (INPA33020); Rio Cuieras just below mouth of rio Bracinho, col. G.T. Prance et al. 14940, 27-IX-1971 (INPA33156); Basin of rio Negro, Tapuruquara, Caatinga, col. G.T. Prance et al. 15631, 28-X-1971 (INPA33848); Foothills of serra Curicuriari, col. G.T. Prance et al. 16165, 6.XI-1971 (INPA34384); Manaus, Reserva Experimental Egler, forest by road side, col. C.C. Berg, E. Lleras \& J. Ramos P17614, 6-XI-1973 (INPA40811); Rio Içana, Nazaré, aldeia dos índios Baniwas, col. M.F. Silva, P. Machado \& O. Pires 1505, 10-V-1973, det. O. Yano (INPA38586); Manaus-Itacoatiara road, $\mathrm{km}$ 69-70, col. G.T. Prance et al. P17527, 5-X-1973 (INPA40723); Manaus, Reserva Florestal Ducke, $\mathrm{km} \mathrm{26,} \mathrm{Manaus-Itacoatiara} \mathrm{road,} \mathrm{col.}$ C.C. Berg et al. P17597, 17598, 6-XI-1973, det. O. Yano (INPA40794, SP147605; INPA40795, SP147606); Rio Cuieras, $2 \mathrm{~km}$ below mouth of rio Bracinho, col. G.T. Prance et al. 17764, 
11-IX-1973, det. O. Yano (INPA41068); Rio Cuieras, $2 \mathrm{~km}$ below mouth of rio Bracinho, col. G.T. Prance et al. 17830, 12-IX-1973 (INPA41134); Manaus-Caracaraí road, km 140, forest by rocky rapids, col. C.C. Berg et al. P18183, 27-IX-1973 (INPA41488): Manaus-Caracaraí road, km 140, col. C.C. Berg et al. P18190, 27-IX-1973 (INPA41495); Reserva Campina, Manaus-Caracaraí road, $\mathrm{km} 62$, col. G.T. Prance et al. 18745, 10-VIII-1973, det. O. Yano (INPA42068); Manaus, estrada do Aleixo, grounds of Compania das Plantações, col. G.T. Prance et al. 18775, 30-VIII-1973 (INPA42254); Manaus-Caracaraí road, km 125. col. C.C. Berg et al. P19482, 12-XI-1973, (INPA42972); Manaus-Caracaraí road, near km 130 area with sandstone rocks along river, col. C.C. Berg et al. P19555, 13-XI-1973 (INPA43039); Manaus-Caracaraí road, near km 130 , area with sandstone rocks along river, col. C.C. Berg et al. P19562, 13-XI-1973, det. O. Yano (INPA43046); Road Manaus-Porto VeIho (BR-319) behind road camp, km 240, col. E. Lleras, C. Holley \& O.P. Monteiro P19579, P19581, 21-XI-1973, det. O. Yario (INPA43063, 43065); Manaus-Porto Velho highway, BR-319, km 268, 28 km South of Igapó Açu, col. G.T. Prance et al. 20653, 20657, 17-III-1974, det. O. Yano (INPA44632, 44637); Mun. de Manaus, Reserva Campina, along the road Manaus-Caracarai, km 60, col. D. Griffin, D.M. Vital \& O. Yano 1, 65, 8-VII-1974, det. O. Yano (SP125222; SP125254); col. D. Griffin, D.M. Vital \& O. Yano 103, 9-VII-1974, det. O. Yano (SP125275); Manaus, Estação Agrícola (CEPLAC), along the road Manaus-Itacoatiara km 30, col. D. Griffin, D.M. Vital \& O. Yano 116, 9-VII-1974 (SP125282); col. D. Griffin, D.M. Vital 154, 11-VII-1974 (SP125300); Manaus, Reserva Ducke, col. D. Griffin, D.M. Vital \& O. Yano 170, 173, 185 e 190, 11-VII-1974 (SP125308, 125310, 125317, 125319); Manaus, ao longo da rodovia Manaus-Itacoatiara, km 12, col. D. Griffin, D.M. Vital \& O. Yano 213, 11-VII-1974 (SP125329); Manaus, Reserva Campina, col. D. Griffin, D.M. Vital \& O. Yano 243, 18-VII-1974 (SP125344); Manaus, Tarumã Alta, col. D. Griffin, D.M. Vital \& O. Yano 272, 23-VII-1974, (SP125356); Manaus, ao longo da rodovia Manaus-Caracarai, km 130, col. D. Griffin, D.M. Vital \& O. Yano 422, 465 e 471, 25-VII-1974 (SP125418, SP125431, SP125432); Manaus, ao longo da rodovia Manaus-Itacoatiara, km 65, Reserva Egler, col. D. Griffin, D.M. Vital \& O. Yano 523, 30-VII-1974 (SP125448); Boa Fé, margem esquerda do rio Negro, col. Eq. Bot. Tropical, 24-VII-1974 (SP125492); Manaus, Rod. Manaus-Caracaraí, km 115, rio Urubu, col. D. Griffin, D.M. Vital \& O. Yano 870 e 911, 13-VIII-1974 (SP125595 e SP125609); Manaus, Campus do INPA, col. D. Griffin, D.M. Vital \& O. Yano INPA31, 26-VII-1974 (SP125663); Manaus, Reserva Florestal Ducke, km 26 da estr. Manaus-Itacoatiara, col. R. Lisboa 24, 40, 48, 173, 12-VIII-1974, det. D. Griffin (INPA55009. SP147635; INPA49101, SP147613; INPA49109, SP147614; INPA55514, SP147646); col. R. Lisboa $84,144,165,13-$ VIII-1974, det. D. Griffin (INPA49146, SP147617; INPA55021, SP147637; INPA55506, SP147644); col. R. Lisboa, 152, 153, 9-VIII-1974, det. D. Griffin (INPA55489, SP147639; INPA55494, SP147640); Manaus, km 130 da estrada Manaus-Caracaraí igarapé de Lages, col. P. Lisboa et al. s/n, 4-XI-1975, det. R. Lisboa (INPA53608, SP147631; INPA53611, SP147632); Rio Javari, margem direita, col. J. Ramos, 15-XI-1976 (INPA56067, SP147648; rio Jaú, afluente do rio Negro, Projeto Radam/Brasil, col. J. Ramos s/n, 24-VIII-1976, det. O. Yano (INPA62269); Estrada Manaus-Itacoatiara, km 26, Reserva Ducke, col. R. Lisboa 191, 29-XI-1976, det. O. Yano (INPA62056, SP47769); Manaus, Acará, Reserva Florestal Ducke, km 26 da estrada Manaus-Itacoatiara, col. P. Lisboa \& R. Lisboa 935, 6-I-1977, det. R. Lisboa (INPA62601, SP47664); Manaus, Reserva Egler, km 70 da estrada Manaus-Itacoatiara, col. R. Lisboa 221, 228, 232, 240, 14-I-1977, det. O. Yano (INPA63732, SP147668; INPA63739, SP147670; INPA63743, SP147671; INPA63751, SP147673); Manaus, Reserva Florestal Ducke, Acará, km 26 da estrada Manaus-Itacoatiara, col. R. Lisboa 255, 279, 28-I-1977, det. R. Lisboa (INPA63766, SP147676; INPA63790, SP147677); Manaus, Reserva Ducke, km 26 da estrada Manaus-Itacoatiara, col. R. Lisboa 350, 351, 354, 5-IV-1977, det. O. Yano (INPA70266, SP147719; INPA70267, SP147720; INPA70270, SP147721); 
rio Jaú, afluente do rio Negro, lago do Paié, margem direita do rio Jaú, col. J. Ramos et al. 715, 716, 717, 6-IV-1977, det. O. Yano (INPA73668, SP147777; INPA73669, SP147778; INPA73670, SP147779); Cordilheira do Pico da Neblina, caminho do rio 31 de Março, à 1.500 $\mathrm{m}$ de alt., perto do acampamento, col. W.A. Rodrigues 9845, 7-XI-1977, det. O. Yano (INPA71504, SP147808); EMBRAPA, km 30 on Manaus-Caracaraí Road, col. W.R. Buck 1752 , 3-XI-1977, det. O. Yano (SP147783); rio Uatumã, lado direito do rio, vegetação de campina. col. P. Ivo S. Braga et al. 3528, 3531, 3534, 3538 , 27-II-1978, det. R. Lisboa (INPA75349, SP147727; INPA75352, SP147728; INPA75355, SP147729; INPA75357, SP147731); estrada Manaus-Itacoatiara, km 52 ramal ZF-1 que liga Manaus-Caracaraí, km 9 col. P. Lisboa et 9!. 1163, 1179, 9-VI-1978, det. O. Yano (INPA76396, SP147774; INPA76411, SP147775). Mato Grosso: Under waterfall at first igarapé after descenting Chapada on Road to Cuiabá, alt. $60 \mathrm{~cm}$, col. G.T. Prance et al. 19334, 19342, 19343, 23-X-1973, det. D. Griffin (INPA42825, 42833, 42834); Gorge of Véu de Noiva, chapada dos Guimarães, col. G.T. Prance et al. 19096, 19097 , 17-X-1973, det. D. Griffin (INPA42587, 42588). Pará: Marajó, Breves, Vila Nova do Aramá, col. P. Cavalcante 1985, 20-IX-1968, det. O. Yano (MG35779); rio Trombetas, vicinity of cachoeira Porteira, col. G.T. Prance et al. 22182, 20-V-1974, det. O. Yano (INPA46118). Território do Amapá: rio Jari, cachoeira das Guaribas, $0^{\circ} 24^{\prime} \mathrm{N}, 53^{\circ} 7^{\prime} \mathrm{W}$, col. W. A. Egler \& A.S. Irwin, 16-VIII-1961, det. O. Yano (INPA46455, IAN11325). Território de Rondônia: rio Pacaás Novos, Primeira Cachuera, col W.D. Reese 13574, 24-III-1978, det. O. Yano (SP147766); rio Pacaás Novos, Primeira Cachuera, serra pe. quena, ca $450 \mathrm{~m}$ alt., col. W.D. Reese 13599, 25-III-1976, det. O. Yano (SP147767); rio Pa. caás Novos, sobre primeira Cachuera. serra pequena, a $500 \mathrm{~m}$, col. W.D. Reese 13349, 15-22-III-1978, det O. Yano (SP147758); col. W.D. Reese 13507, 13508, 20-III-1978, det. O. Yano (SP147762; INPA). Territóric de Roraima: serra"dos Surucucus $2^{\circ} 42-47^{\prime} \mathrm{N}, 63^{\circ} 33-36^{\prime} \mathrm{W}, \mathrm{NE}$ of Mission Station, $1000-1400 \mathrm{~m}$ alt., col. G.T. Prance et al. 10006, 17-II-1969 (INPA27139);
Maitá Mountains $3^{\circ} 15^{\prime} \mathrm{N}, 63^{\circ} 28^{\prime} \mathrm{W}$, col. G.T. Prance et al. 13640, 17-II-1971, det. O. Yano (INPA28789); Auaris Mission, rio Auaris, alt. $2600 \mathrm{ft}$, Campina forest, col. G.T. Prance, T. Dobzhansky \& J. Ramos 20047, 6-XII-1973, det. D. Griffin (INPA43972); Vicinity Auaris $64^{\circ} 25^{\prime} \mathrm{W}$, $4^{\circ} 6^{\prime} \mathrm{N}, 800 \mathrm{~m}$ alt., col. G.T. Prance et al. 21489 , 28-VII-1974, det. D. Griffin (INPA45434); serra Parima, col. Bruce Nelson 21499, 28-VII-1974 (INPA, SP125539); Acampamento do 6. BECJundiaí, km 328 on Manaus-Caracaraí road, from junction Manaus-Itacoatiara Road, col. W.R. Buck 1801, 16-XI-1977, det. O. Yano (SP147787); acampamento Novo Paraíso, at $\mathrm{km} \mathrm{551}$, along Manaus-Caracarai Road, $10 \mathrm{~km}$ $\mathrm{N}$ of rio Amana, col. W.R. Buck 1903, 21-XI-1977, det. O. Yano (SP147796); Boca da Mata, $216 \mathrm{~km}$ of Boa Vista, on Boa Vista-Caracas Road, col. W.R. Buck 1893, 1993, 2019, 2026, 30-XI-1977 det. O. Yano (SP147801; SP147802; SP147804; INPA). L. martianum é a mais comumente encontrada em: qualquer tipo de substrato formando densos tapetes, às vezes apresentam uma coloração violácea. Nas regiōes de mata úmidas formam extensos tapetes. Possuem os filídios fortemente facados e quando em corte transversal na base, os clorocísticos na parte ventral e as células leucocísticas uma concavidade.

Ochrobryum gardneri (C. Muell.) Lindb. Mato Grosso: Vicinity of Co'égio Bur.ti, chapada dos Guimarães, disturbed dry forest, alt. $720 \mathrm{~m}$, col. G.T. Prance et al. 19015, 19016, 15-X-1973, det. D. Griffin (INPA42506, 42507); Gorge of Véu de Noiva, chapada dos Guimarães, col. G.T. Prance et al. 19149, 17-X-1973, det. D. Griffin (INPA42640); Véu de Noiva, chapada dos Guimarães, alt. $720 \mathrm{~m}$, col. G.T. Prance et al. 19398, 25-X-1973, det. D. Griffin (INPA42889); Mun. de Aripuanã, Dardanelos antiga estrada do aeroporto, col. P. Lisboa et 507, 3-X-1975, det. D. Griffin (INPA52720, SP147622); Mun. de Aripuanã, Est. Humboldt, col. C. Damião 2456, 2474, 26-III-1977, det. O. Yano INPA69482, SP147703; INPA69500, SP147711); Mun. de Aripuanã, Est. Humboldt, col. C. Damião 2465, 18-IV-1977, det. O. Yano (INPA69491, SP147704). Território de Rondônia: Vicinity of Santa Barbara, $15 \mathrm{~km}$ east of $\mathrm{km}$ 
117, col. G.T. Prance \& J.F. Ramos 6910, 14-VIII-1968 (INPA23669); rio Pacaás Novos, sobre primeira Cachuera, col. W.DD. Reese 13488, 20-III-1978, det. O. Yano (SP147761). O. gardenri apresenta uma coloração verde-acinzentada, crescem formando tapetes em rochas, em troncos de árvores vivas ou mortas e em madeiras. Apresentam geralmente gemas globosas no ápice do filídio; a margem do filídio com 3-6 fileiras de células.

Ochrobryum obtusifolium (C. Muell.) Mitt. Mato Grosso: $5 \mathrm{~km} \mathrm{E}$ of town of chapada dos Guimarães on road to EMBRATEL, disturbed forest on South facing of slope of chapada, alt. $720 \mathrm{~m}$, col. G.T. Prance et al. 19379, 24-X-1973, det. O. Yano (INPA.42870); Mun. de Aripuanã, Dardanelos, col. P. Lisboa et al. 508, Aripuanã, Dardanelos, col. P. Lisboa et al. 508, 30-IX-1975, det. O. Yano (INPA52750, SP147623); Mun. de Aripuanã, col. C. Damião 775, 18-II-1977, det. O. Yano (INPA66220, SP147694); Município de Aripuanã, estação Humbold, col. C. Damião 2450, 28-III-1977, det. O. Yano (INPA69476, SP147701). O. obtusifolium possui o ápice do filídio obtuso; a margem com 3-4 fileiras de células e o opérculo curto. Esta espécie é a primeira vez citada para a região amazônica. Crescem em rochas úmidas, troncos de árvores vivas ou mortas.

Ochrobryum stenophyllum Besch. Acre: $33 \mathrm{~km}$ de Rio Branco, em estrada à Brasileira, col. W.R. Reese 13263, 26-II-1978, det. O. Yano (INPA, SP147756). Mato Grosso: Mun. de Aripuanã, col. C. Damião 761, 14-II-1977, det. O. Yano (INPA66206, SP147688); Mun. de Aripuanã, Estação Humboldt, coletado na beira da Cachoeira, col. C. Damião 2424, 2478 , 26-IV-1977, det. O. Yano (INPA69446, SP147700; INPA69504, SP147715); Estação Humboldt, col. C. Damião 2467, 17-IV-1977, det. O. Yano (INPA69493, SP147706). Pará: serra do Cachimbo, floresta de $30 \mathrm{~m}$ ao longo da BR-163, km 845 , col. E. Lleras \& J.H. Kirkbride 1182, 18-II-1977, det. O. Yano (INPA64336). Território de Rondônia: rio Pacaás Novos, Primeira Cachuera, serra pequena, ca 450 al alt.. col. W.D. Reese̊ 13603, 25-III-1978, det. O. Yano (SP147768). Esta espécie possui a cor verdeamarelada. Cresce em pequenos tufos em ma- deira em decomposição e troncos de árvore. A margem do filídio com 6 fileiras de células longo-retangulares.

Octoblepharum africanum (Broth.) Card Amazonas: Manaus, ao longo da rodovia $\mathrm{Ma}$ naus-Caracaraí, km 130, col. D. Griffin, D.M. Vital \& O. Yano 368, 25-VII-1974, det. O. Yano (SP125399). Território de Roraima: serra Parima, col. Bruce Nelson P21483, 28-VII-1974, det O. Yano (SP125538). O. africanum é a primeira vez citada para a região. Crescem em rochas arenosas e na base dos troncos de árvores. Esta espécie difere de todas as outras pela seção transversal do filídio que é quase um semicírculo, e as células clorocísticas estão no meio da costa. Esporófito latera! ou terminal.

Octoblepharum albidum Hedw. Acre: Cruzeiro do Sul, vicinity of Porangaba, rio JuruáMirim, col. Maas et al. P13052, 17-V-1971 (INPA31416); Cruzeiro do Sul, sub-base do Projeto Radam/Brasil, col. J. Ramos \& G. Mota 232, 2-III-1976, det. O. Yano (INPA56897, SP147653); 15 km E de Rio Branco em estrada à Abuná, col. W.D. Reese 13148, 24-II-1978, det. O. Yano (INPA; $15 \mathrm{~km} \mathrm{E}$ de Rio Branco em estrada à Abuná, col. W.D Reese 13162, 24-II-1978, det. O. Yano (INPA); $35 \mathrm{~km} \mathrm{~S}$ of Rio Branco, em estrada à Brasiléia, col. W.D. Reese 13266, 26-II-1978, det. O. Yano (INPA); $63 \mathrm{~km}$ $S$ of Rio Branco, em estrada à Brasiléa, co! . W.D. Reese 13278, 26-II-1978, det. O. Yano (INPA). Amazonas: Vicinity of Manaus, Ponta Negra, col. G.T. Prance et al. 11704, 31-III-1971 (INPA30016); Manaus, Reserva Florestal Ducke, km 27 Manaus-ltacoatiara rnad, col. G.T. Prance et al. 11308, 2-IV-1971, (NY, INPA29708, SP147603); Manaus, cachoeira Baixa Tarumã, col. G.T. Prance et al. 11672, 31-III-1971 (INPA29984); Manaus, estrada Mauá, igapó col. G.T. Prance et al. 11732, 5-IV-1971 (INPA30044); rio Purus, lago Preto, $2 \mathrm{~km}$ No:th of Lábrea, col. G.T. Prance et al. 13734, 26-VI-1971 (INPA31950); Reserva Experimental do INPA, Manaus-Caracaraí road, km 61, col. G.T. Prance et al. 18709, 3-VIII-1973 (INPA42022); Reserva Ducke, km 26 Manaus-Itacoatiara road, col. C.C. Berg et al. P17602, 6-XI-1973, det. O. Yano (INPA40799, SP147607); rio Cuieras, 2 
$\mathrm{km}$ below mouth of rio Bracinho, col. G.T Prance et al. 17899, 17900, 13-|X-1973, det. O. Yano (INPA41203, 41204); Manaus-Caracaraí

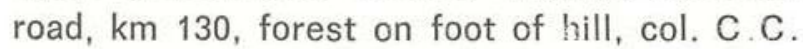
Berg et al. 19533, 13-Xl-1973, det. O. Yano (INPA); Manaus, Reserva Florestal Ducke, along the road Manaus Itacoatiara, km 26, col. D. Griffin, D. M. Vital \& O. Yano 104, 9-VII-1974 (SP125276); Manaus, Estação Agrícola (CEPLAC), along the road Manaus-Itacoatiara, km 30, col. D. Griffin, D.M. Vital \& O. Yano 157, 11-VII-1974 (SP125301); Manaus, ao longo da rodovia Manaus-Itacuatiara, $\mathrm{km} \mathrm{12,}$ col. D. Griffin, D.M. Vital \& O. Yano 222, 11-VII-1974 (SP125335); Manaus, Tarumã, col. D. Griffin, D.M. Vital \& O. Yano 220, 14-VII-1974 (SP125339); Manaus, estrada des Franceses, km 5, Balneário do SESC, co!. O. Yano (261), 21-VII-1974 (SP125349); Manaus, Ponta Negra, col. D. Griffin, D.M. Vital \& O. Yano 285, 23-VII-1974 (SP125366); Manaus, rodovia Manaus-Caracaraí, km 36, col. D. Griffin, D.M. Vital \& O. Yano 601, 1-Vlil-1974 (SP125478; Boa Fé, margem esquerda do rio Negro, rio Apuaú, col. Eq. Bot. Tropical, (607, 615, 626, 627), 24-VII-1974 (SP125484; SP125470; SP125497; SP125498); Manaus, Praça Roosevelt, col. D. Griffin, D. M. Vital \& O. Yano 830, 12-VIII-1974 (SP125583); Manaus, rodovia Manaus-Caracaraí, $\mathrm{km}$ 115, rio Urubu, col. D. Griffin, D.M. Vital \& O. Yano 894, 13-VIII-1974 (SP125605); Manaus, Campus do INPA, col. D. Griffin, D.M. Vital \& O. Yano 13, 34, 26-VII-1974 (SP125658; SP125665); Manaus, Reserva Florestal Ducke, km 26 da estrada Manaus-Itacoatiara, col. P. Lisboa 34, 9-VIII-1974, det. O. Yano (INPA49095, SP147611); Reserva Florestal Ducke, km 26 da estrada Manaus-Itacoatiara, col. P. Lisboa 81, 13-VIII-1974, det. O. Yano (INPA49142, SP147616, mixed O. cylindricum); Manaus, Reserva Florestal Ducke, km 26 da estrada Manaus-Itacoatiara, col. R. Lisboa, 157, 9-VIII-1974, det. D. Griffin (INPA55498, SP147642); Manaus estrada do Aleixo, $\mathrm{km} \mathrm{4}$, Campus do INPA, col. P. Lisboa 186, 12-IX-1975, det. O. Yano (INPA51441); Manaus, aeroporto supersônico, estrada Manaus-Itacoatiara, col. P. Lisboa 249, 21-IX-1975, det. O. Yano (INPA51718,
SP147619); rio Javari, margem direita, col. J. Ramos, 15-XI-1975, det. O. Yano (INPA54065, SP147633); Reserva Egler, km 70 da estrada Manaus-Itacoatiara, col. R. Lisboa 222, 235, 14-I-1977, det. O. Yano (INPA63733, SP147669; INPA63747, SP147762); Reserva Florestal Duc$\mathrm{ke}, \mathrm{km} 26$ da estrada Manaus-Itacoatiara, Acará, col. R. Lisboa 293, 28-I-1977, det. O. Yano (INPA63804, SP147678, mixed $O$. pulvinatum); Reserva Ducke, km 26 da estrada Manaus-lta. coatiara, col. R. Lisboa 317, 318, 320, 323, 332, 337, 11-III-1977, det. O. Yano (INPA63828, SP147680; INPA63829, SP147681; INPA63831 SP147682; INPA63834, SP147683; INPA63843, SP147685; INPA63848, SP147687); col. R. Lisboa 343, 5-IV-1977, det. O. Yano (INPA70259, SP147716); col. P. Lisboa \& R. Lisboa 902, 923, 6-1-1977, det. O. Yano (INPA62568, SP147658; INPA.62589, SP147663); estrada Manaus-Caracaraí, $\mathrm{km} \mathrm{61}$, ramal à esquerda em frente a Reserva Biológica de Campina, col. P. Lisboa \& O.P. Monteiro 964, 11-I-1977, det. O. Yano (INPA62722, SP147667); Tarumã Grande, along rio Tarumã, white sand igarpó, col. W.R. Buck 1795, 9-XI-1977, det. O. Yano (NY, SP147786); Manaus, cidade, col. W.D. Reese 12678, 6-12-1-1978, det. O. Yano (INPA). Mato Grosso: Aripuanã, near Humboldt Center, on road to new airport, $59^{\circ} 21^{\prime} \mathrm{N}, 10^{\circ} 12^{\prime} \mathrm{S}$, col. E. Lleras \& J.F. Lima, P18276, 9-X-1973, det. O. Yano (INPA41581); Margin of new airstrip of Humboldt Center, rio Aripuanã, $59^{\circ} 21^{\prime} \mathrm{N}$, $10^{\circ} 12$ 'S, col. G.T. Prance et al. 18339, 18347, 18366, 10-X-1973 (INPA41641; 41649; 41668); Mun. de Aripuanã, Dardanelos, picada seguindo o aeroporto, col. P. Lisboa et al. 382, 359, 28-IX-1975, det. R. Lisboa \& D. Griffin (INPA52846, SP147627; INPA52872, SP147628); floresta da antiga estrada do aeroporto, col. P. Lisboa et al. 513, 30-IX-1975, det. O. Yano (INPA52905, SP147629 mixed Ochrobryum obstusifolium); Município de Aripuanã, Estação Humboldt, col. C. Damião 2451, 2470 , 28-III-1977, det. O. Yano (INPA69477, SP147702; INPA69496, SP147709); col. C. Damião 2466, 28-III-1977, det. O. Yano (INPA69492, SP147705). Pará: Belém, Aurá, col. J.M. Pires 4648, 1-V-1954, det. O. Yano 22-XII-1980 (IAN82442). Faz. Tuiuiu, rio Arari, ilha de Marajó, col. 
P. Ledoux 185, 23-IX-1952, det. O. Yano (IAN84870); campo do Caembê, Mun. da Vigia col. G.A. Black \& L.B. Smith 56-18822, 12-X-1956, det. O. Yano (IAN95323); alto Tapajós, rio Cururu, Bernardino, col. W.A. Egler 1004, 23-VII-1959, det. O. Yano (MG23806); alto Tapajós, rio Cururu, Missão, col. W.A. Egler 1320, 12-11-1960 (MG 24367); Oriximiná, estrada para a gruta, col. M. Silva 1176, 15-I-1968 (MG 35272, SP110327); serra do Cachimbo, BR-163, campina de areia branca, km 832, col. E. Lleras \& J.H. Kirkbride 1174, 16-II-1977, det. O. Yano (INPA64328); serra do Cachimbo, floresta de $30 \mathrm{~m}$ ao longo da BR-163, $\mathrm{km} 845$, col. E. Lleras \& J.H. Kirkbride 1179, 18-II-1977, det. O. Yano (INPA64333); Jubin, a $6 \mathrm{~km}$ de Salvaterra a $1500 \mathrm{~m}$ da Raia, col. P. Lisboa \& R. Lisboa 1181, 13-VII-1978, det. O. Yano (INPA78219. SP147776); Acará, Boa Vista, col. R.S. Secco et al. 33, 32, 18-IV-1979, det. O. Yano (MG); col. R.S. Secco et al. 31, 18-IV-1979 (MG), Mun. de Santarém, localidade Sertão, margem esquerda do rio Curuá, col. R.L. Fróes 33088-XVIII, nov. 1955, det. O. Yano (IAN94795). Território de Amapá: Estrada Macapá-Clevelândia, km 130, col. G.A. Black 55-18598, 28-VIII-1975, det. O. Yano (IAN89505). Território de Rondônia: rio Jamari, Santa Cruz, col. J.M. Pires 9993, 29-VI-1965, det. O. Yano (IAN); Track between Mutumparaná and rio Madeira, col. G. T. Prance et al. 5511, 3-VII-1968 (INPA22271); Vicinity of Santa Barbara, $15 \mathrm{~km}$ East of km 117, col. G.T. Prance \& J.F. Ramos 6933, 14-VIII-1968, det. O. Yano (INPA23692); Secondary vegetation, col. P.J.M. \& H. Maas 404, 20-VIII-1971, det. O. Yano (INPA34954); On km 240 from Porto Velho to Rondônia, col. E. Lleras et al. P19433, 30-X-1973, det. O. Yano (INPA42923); Guajará-Mirim, km 20 da estrada Saldanha, col. E. Lleras et al. 1104, 12-I-1977, det. O. Yano (INPA63403); rio Pacaás Novos, sobre primeira Cachuera, col. W.D. Reese 13473, 19-III-1978, det. O. Yano (INPA). Território de Roraima: Base of serra Tepequem, Boca da mata, col. G.T. Prance et al. 4334, 11-II-1967 (INPA19969); Lower slopes of serra da Lua $\left(2^{\circ} 25-29^{\prime} \mathrm{N}, 60^{\circ} 11-14^{\prime} \mathrm{W}\right)$, by igarape Camarão, col. G.T. Prance et al. 9376, 23-I-1969, det. O. Yano (INPA26512); Vi- cinity of Uaicá airstrip, rio Uraricoeira $3^{\circ} 33^{\prime} \mathrm{N}$, $63^{\circ} 11^{\prime} \mathrm{W}$, col. G.T. Prance et al. 10763, 28-II-1971, det. O. Yano (INPA29115); Vicinity of Mucajaí airstrip, col. G.T. Prance et al. 10952, 14-III-1971 (INPA29354); rodovia Caracaraí-Boa Vista, km 13, col. Pedro N. Conceição (650), 2-VIII-1974, det. O. Yano (SP125517); Vicinity of Auaris, $64^{\circ} 25^{\prime} \mathrm{W}, 4^{\circ} 6^{\prime} \mathrm{N}, 800 \mathrm{~m}$ alt., col. G.T. Prance et al. 21343, 21344, 23-VII-1974, det. O. Yano (INPA45319; 45320); Vicinity of Auaris, col. G.T. Prance et al. 21337, 23-VII-1974, det. O. Yano (INPA45313); Acampamento do $6 .^{\circ}$ BEC-Jundiá, km 328 on Manaus-Caracaraí road, from junction at Manaus-Itacoatiara road, col. W.R. Buck 1860 , 17-XI-1977, det. O. Yano (SP147792); Boca da Mata, $216 \mathrm{~km} \mathrm{~N}$ of Boa Vista on Boa Vista-Caracas road, col. W.R. Buck 1962, 1959, 29-XI-1977, det. O. Yano (SP147799). É a espécie relativamente comum na região amazônica. É facilmente reconhecido pela presença de cápsula ovóide e com menos que $1 \mathrm{~cm}$ compr. e o ápice do filídio obtuso e denteado. Esta espécie apresenta uma distribuição cosmopolita, cresce em qualquer tipo de substrato.

Octoblepharum albidum Hedw. var. violascens C. Muell. Acre: Cruzeiro do Sul, nos arredores do acampamento, próximo do aeroporto Novo, col. O.P. Monteiro \& C. Damião 176, 7-II-1976, det. O. Yano (INPA56015, SP147647. Amazonas: Manaus, Reserva Florestal Ducke, col. P. Lisboa 50, 12-VIII-1974, det. O. Yano (INPA49111, SP147615); Manaus, Reserva Florestal Ducke, km 26 da estrada Manaus-Itacoatiara, col. R. Lisboa 151, 154, 9-VIII-1974, det. O. Yano (INPA55488, SP147638; INPA55495, SP147641). Mato Grosso: Gorge of Véu de Noiva, chapada dos Guimarães, col. G.T. Prance et al. 19150, 17-X-1973, det. O. Yano (INPA42641); Mun. de Aripuanã, col. P. Lisboa et al. 609, 3-X-1975, det. O. Yano (INPA52656, SP147620); Mun. de Aripuanã, estação Humboldt, col. C. Damião 2468, 17-IV-1977, det. O. Yano (INPA69494, SP147707). Território de Rondônia: Porto Velho to Cuiabá highway, vicinity of Santa Barbara, $15 \mathrm{~km}$ East of $\mathrm{km}$ 117, col. G.T. Prance \& J.F. Ramos 6927, 14-VIII-1968, det. O. Yano (INPA23686); 
Basin of rio Madeira, km 90-93 Madeira-Mamoré railroad, near Jaciparaná, col. G.T. Prance et al. 5408, 30-VI-1968 (INPA22168); GuajaráMirim, km 20 da estrada Saldanha, col. E Lleras et al. 1106, 12-I-1977, det. O. Yano (INPA63405). É a primeira vez citada para a região amazônica. Apresenta semelhança a $O$. albidum, mas a base dos filídios são fortemente violáceos ou avermelhados. Crescem em casca de árvore pobre, sobre palmeiras e rochas secas.

Octoblepharum ampullaceum Mitt. Amazonas: Basin of rio Negro, Summits of serra Curicuriari, col. G.T. Prance et al. 16101 5-XI-1971 (INPA34320); road Manaus-Caracaraí, km 125, col. C.C. Berg et al. P19489, 12-XI-1973, det. O. Yano (INPA42979); Manaus, Reserva Campina along the road Manaus-Caracaraí, km 61, col. D. Griffin, D.M. Vital \& O. Yano 36, 37, 8-VII-1974, det. O. Yano (SP125241; SP125242, mixed Micropterygium); Manaus, ao longo da rodovia Manaus-Caracaraí, km 130, col. D. Griffin, D.M. Vital \& O. Yano 398, 25-VII-1974, (SP12411); Manaus, rodovia Manaus-Caracaraí $\mathrm{km}$ 61, Reserva Campina, col. D. Griffin, D.M. Vital \& O. Yano 538, 1-VIII-1974 (SP125452); Manaus, igarapé Lajes, col. D. M. Vital 4320, 6-VIII-1974 (SP125549); Manaus, Reserva Florestal Ducke, km 26 da estrada Manaus-Itacoatiara, col. P. Lisboa 31, 85, 8-VIII-1974, det. O. Yano (INPA49093, SP147610; INPA48147, SP147618); Manaus, Reserva Biológica Campina, estrada Manaus-Caracaraí, km 62, col. R. Lisboa 43, 13-XI-1974, det. D. Griffin (INPA48826); Manaus, Reserva Florestal Ducke, igarapé Acará, km 26 da estrada Manaus-Itacoatiara, col. P. Lisboa 908, 6-I-1977, det. O. Yano (INPA62574, SP147659); Manaus, Reserva Egler km 70 da estrada Manaus-Itacoatiara, coI. R. Lisboa, 252, 14-I-1977, det. O. Yano (INPA63763, SP146674). Pará: serra do Cachimbo, BR-163, Campina de areia branca, $\mathrm{km} \mathrm{832,} \mathrm{col.} \mathrm{E.} \mathrm{L!eras} \mathrm{\&}$ J.H. Kirkbride 1173, 16-II-1977, det O. Yano (INPA64327). Território de Roraima: Vicin'ty of Auaris, $64^{\circ} 25^{\prime} \mathrm{W}, 4^{\circ} 6^{\prime} \mathrm{N}, 800 \mathrm{~m}$ alt., col. G.T. Prapce et al. 21483, 28-VII-1974, det. O. Yano (INPA45428). Ocorre principalmente em rochas, nas areias próximo as rochas, sobre raízes, troncos de árvores vivas ou em decomposição, em tronco de palmeira. A espécie apresenta alguma semelhança com $O$. stramineum, mas os filídios são mais delgados e o corte transversal na região medianas é \pm um triângulo equilátero com os bordos arredondados.

Octoblepharum cocuinse Mitt. Acre: serra da Lua, rio Urubu between cachoeira Iracema and Natal, col. G.T. Prance et al. 4996, 6-VI-1968 (INPA21756, NY, SP147774); Cruzeiro do Sul, rio Juruá \& rio Moa, $6 \mathrm{~km}$ above school, col. G.T. Prance et al. 12457, 12459, 25-IV-1971, det. O. Yano (INPA30780; 30782). Amazonas: Manaus, Reserva Florestal Ducke, km $27 \mathrm{Ma}$ naus-Itacoatiara road, col. G.T. Prance et 31 . 11299, 2-IV-1971 (INPA29699); Manaus estrada Mauá, igapó, col. G.T. Prance et al. 11730, 5-IV-1971 (INPA30042); Fortaleza Savana, rio Puciari tributary of rio Ituxi, $20 \mathrm{~km}$ above mouth, col. G.T. Prance et al. 13842, 1-VII-1971 (INPA32058); rio Curuquetê, vicinity of cachoeira São Bento, col. G.T. Prance et al. 14334, 15-VII-1971 (INPA32550); basin of rio Negro, rio Cuieras just below mouth of rio Bracinho, col. G.T. Prance et al. 14868, 25-IX-1971 (INPA33084); basin rio Negro, rio Uneiuxi, 100-200 km above mouth, col. G.T. Prance et al. 15510, 21-X-1971 (INPA33727); Manaus, Tarumã Alto, in tufs on dripping clifs mixed Selaginella sp, col. D. Griffin, D.M. Vital \& O. Yano 264, 23-VII-1974 (SP125351); ao longo da rodovia Manaus-Caracaraí, km 130. entrada da caverna, col. D. Griffin, D.M. Vital \& O. Yano 440, 25-VII-1974 (SP125424); paredão escuro da gruta, col. D. Griffin, D.M. Vital \& O. Yano 467, 25-VII-1974 (SP125432); Manaus, rodovia Manaus-Caracaraí, km 115, rio Urubu, col. D. Griffin, D.M. Vital \& O. Yano 878, 879, 881, 882, 13-VIII-1974, det. O. Yano (SP125598; SP125599; SP125601; SP125602); col. D. Griffin, D.M. Vital \& O. Yano, 928, 929, 13-VIII-1974, det. O. Yano (SP125614; SP125615); Manaus, Campus do INPA, col. D. Griffin, D.M. Vital \& O. Yano INPA35, 26-VII-1974, det. O. Yano (SP125666); Manaus, Reserva Florestal Ducke, igarapé Acará, km 26 da estrada Manaus-Itacoatiara, col. P. Lisboa \& R. Lisboa 911, 6 I-1977, det. O. Yano 
(INPA62577, SP147660). Mato Grosso: Under waterfall at first igarapé after descending chapada on road to Cuiabá, alt. $600 \mathrm{~m}$, col. G.T. Prance et al. 19333, 23-X-1973 (INPA42824). Esta espécie geralmente apresenta cor averm?Ihada, são ramificados, e o filídio muito quebradiço e o seu ápice agudo ou ligeiramente acuminado, algumas vezes apiculado e ondulado. Cresce nas fendas das rochas, no solo arenoso, nos troncos de árvore viva, em decomposição, na base e tronco de palmeira e em rochas encharcadas.

Octoblepharum cylindricum Mont. Amazonas: vicinity of Manaus, $\mathrm{km} 12$ Manaus-Caracaraí road, col. G.T. Prance et al. 11365, 5-IV-1971 (INPA29765); Manaus, cachoeira Baixa Tarumã, col. G.T. Prance et al. 11673, 31-III-1971 (INPA29985); rio Negro, 10-15 km North of serra Jacamim, col. G.T. Prance et al. 15662, 15663, 29-X-1971, det. O. Yano (INPA33879, NY, SP148773; INPA33880); INPA, estrada do Aleixo, $\mathrm{km} \mathrm{3}$, near tracking station col. W.C. Steward \& J.F. Ramos, P20127, 26-XII-1973, det. O. Yano (INPA44050); Manaus, Reserva Campina, along the road Manaus-Caracaraí, km 61, col. D. Griffin, D.M. Vital \& O. Yano 35, 8-VII-1974 (SP125240); Manaus, Reserva Ducke, col. D. Griffin, D.M. Vital \& O. Yano 165, 11-VII-1974 (SP125306); ao longo da rodovia Manaus-Itacoatiara, $\mathrm{km} \mathrm{12.} \mathrm{col.} \mathrm{D.}$ Griffin, D.M. Vital \& O. Yano 224, 11-VII-1974 (SP125337); Manaus, estrada dos Franceses $\mathrm{km} \mathrm{5}$, Balneário do SEC, col. O. Yano (255), 21-VII-1974 (SP125348); Manaus, ao longo da rodovia Manaus-Caracaraí, km 130, D. Griffin, D.M. Vital \& O. Yano 384, 25-VII-1974 (SP125405); ao longo da rodovia Manaus-Caracaraí, km 130, col. D. Griffin, D.M. Vital \& O. Yano 439, 25-VII-1974 (SP125428); Boa Fé, margem esquerda do rio Negro, rio Apuaú, col. Eq. Botânica tropical (630), 27-VII-1974, det. D. Griffin (SP125501); Manaus, rodovia ManausCaracaraí, km 60, Reserva Campina, col. D. Griffin, D.M. Vital \& O. Yano 964, 15.VIII-1974 (SP125631); Manaus, Campus do INPA, col. D. Griffin, D.M. Vital \& O. Yano INPA12, 33, 26-VII-1974 (SR125657; SP125664); Manaus, Reserva Biológica Campina, estrada Manaus-Caracaraí, km 62, col. R. Lisboa, 33, 13-XI-1974, det. D. Griffin (INPA48816); col. R. Lisboa 51, 21-XI-1974, det. D. Griffin (INPA48834); col. R. Lisboa 63, 28-XI-1974, det. D. Griffin (INPA48846); Manaus, Reserva Florestal Ducke, km 26 da estrada Manaus-Itacoatiara, col. R. Lisboa 161, 8-VIII-1974, det. O. Yano (INPA55502, SP147643); col. R. Lisboa 168, 13-VIII-1974, det. D. Griffin (INPA55509, SP147645); Manaus, Campina de Ponta Negra, terreno do Sr. T. Loureiro, col. R. Lisboa 297. 18-II-1977, det. R. Lisboa (INPA63808, SP14779); Manaus, Reserva Ducke, km 26 da estrada Manaus-Itacoatiara, col. R. Lisboa 346, 5-IV-1977, det. O. Yano (INPA70262, SP147718); rio Cueiras, col. P. Lisboa 824, 5-III-1976, det. O. Yano (INPA54940, SP147634); rio Uatumã, lado direito do rio, col. P. Ivo S. Braga \& O. Lisboa et al. 3536, 27-II-1978, det. O. Yano (INPA75356, SP147730). Mato Grosso: $5 \mathrm{~km} \mathrm{E}$ of town of chapada dos Guimarães on road EMBRATEL, disturbed forest on South facing slope of chapada, alt. 720m, col. G.T. Prance et al. 19382 , 24-X-1973, det. D. Griffin (INPA42873); Mun. de Aripuanã, Estação Humboldt, próxima a cachoeira, col. C. Damião 2477, 26-III-1977, det. R. Lisboa (INPA69503, SP147714). Pará: Regiāo Cametá, Campina a $2 \mathrm{~km}$ da beira do rio Vizeu, col. E. Oliveira 1310, 7-II-1961 (IAN111150); Basin of rio Trombetas, $25 \mathrm{~km}$ up the rio Mapueira, col. D.G. Campbell et al. P22559, 7-VI-1974, det. R. Lisboa (INPA46557, NY, SP148772); Rodovia Cuiabá-Santarém, BR-163, córrego São Bento, col. E. Lleras \& J.H. Kirkbride 1189, 21-II-1977 det. O. Yano (INPA64343). Território de Rondônia: Serra dos Murales, 14 km NNW of junction of rio Madeira and Abunã, col. G.T. Prance et al. 6024, 14-VII-1968 (INPA22780); rio Pacaás Novos, Primeira Cachuera, col. W.D. Reese 13302, 14-11l-1978, det. O. Yano (SP147757); rio Pacaás Novos, $230 \mathrm{~m}$ alt., col. W.D. Reese 13678, 27-III-1978, det. O. Yano (INPA). Território de Roraima: Rio Uraricoera, vicinity of Uaicá airstrip, col. G.T. Prance et al. 19955, 2-XII-1973, det. O. Yano (INPA43883). Esta espécie cresce em densos coxins nos troncos de árvores vivas, em decomposição, queimados, em troncos de palmeiras, no solo humoso e muito comum no solo arenoso de campinarana, sobre rochas 
graríticas úmidas. Reconhece-se facilmente pelo comprimento da seta ( $\pm 3 \mathrm{~cm}$ compr), cápsula cilindrica e também pelo ápice do filídio geralmente inteiro.

Octoblepharum erectifolium Mitt. ex Williams. Amazonas: Manaus, Road Manaus-Caracaraí, $\mathrm{km} \mathrm{115}$, rio Urubu, col. D. Griffin, D.M. Vital \& O. Yano 930, 13-VIII-1974, det. O. Yano (SP125612). Território de Rondônia: rio Pacaás Novos, col. W.D. Reese 13658, 27-III-1978, det. O. Yano (INPA). O. erectifolium é a $1 .^{\circ}$ citação para a região amazônica. Reconhece-se facilmente das outras espécies pelas folhas finas e muito longas, e são muito delicadas. Crescem geralmente em rochas e local bem sombrio.

Octoblepharum pellucidum C. Muell. Amazonas: Manaus, Reserva Campina along the road Manaus-Caracaraí, km 61, col. D. Griffin, D.M. Vital \& O. Yano 61, 72, 8-VII-1974, det. O. Yano (SP125250; SP125258); Manaus, ao longo da rodovia Manaus-Caracaraí, km 130, col. D. Griffin, D.M. Vital \& O. Yano 428, 25-VII-1974, det. O. Yano (SP125420). Mato Grosso: Véu de Noiva, chapada dos Guimarães, alt. $720 \mathrm{~m}$, col. G.T. Prance et al. 19407, 25-X-1973, det. O. Yano (INPA42898). Pará: Belém, col. P. Ledoux 151, 23-X-1952, det. O. Yano (IAN); Maraió, Breves, Vila Nova do Aramá, col. P. Cavalcante 1973, 20-IX-1968, det. O. Yano (MG 35767); Paragominas, faz. Acatauassú, col. R.R. Santos 50, 27-III-1974, det. O. Yano (IAN147880). Território de Rondônia: rio Pacaás Novos, serra pequena, ca $450 \mathrm{~m}$ alt. col. W.D. Reese 13602, 25-III-1978, det. O. Yano (INPA). Território de Roraima: Acampamento do 6. BEC. Jundiá, km 328 on Manaus Caracaraí road, from junction at Manaus-Itacoatiara road, col. W.R. Buck 1816, 1821, 16-XI-1977, det. O. Yano (SP147788; INPA); col. W.R. Buck 1822, 16-XI-1977, det. O. Yano (SP147789); acampamento Novo Paraíso at km 551, along ManausCaracaraí road, $10 \mathrm{~km}$ of rio Amaná, col. W.R. Buck 1888, 21-XI-1977, det. O. Yano (SP147795). O. pellucidum possui as folhas bem delgadas $€$ o ápice denticulado. Cresce nos troncos de árvores vivas, em palmeiras e rochas arenosas.
Octoblepharum pulvinatum (Dozy et Molk.) Mitt. Acre: Cruzeiro do Sul, estrada Alemanha, col. G.T. Prance et al. 11904, 14-IV-1971 (INPA30215); Cruzeiro do Sul, rio Juruá \& rio Moa, slopes of serra da Moa, col. G.T. Prance et al. 12130, 19-IV-1971 (INPA30450); Cruzeiro do Sul, sub-base do aeroporto, Projeto Radam/Brasil, col. J. Ramos \& G. Mota 340, 6-III-1976, det. O. Yano (INPA57013, SP147654); col. J. Ramos \& G. Mota 366, 8-III-1976, det. O. Yano (INPA57175, SP147655). Amazcnas: Humaitá, rio Madeira, road Humaitá to Lábrea km 83, col. G.T. Prance et al. 3272, 24-XI-1966, det. O. Yano (INPA18848); Vicinity of Manaus, Ponta Negra, col. G.T. Prance et al. 11695, 11708, 31-III-1971 (INPA30007; 30020); Manaus. Reserva Florestal Ducke, km 27 Manaus-Itacoatiara road, col. G.T. Prance et al. 11299, 11306, 2-IV-1971, det. O. Yano (INPA29699, SP147600; INPA29706, SP147602); Ponta Negra, col. G.T. Prance et al. 11691, 11710, 31-III-1971 (INPA30003; INPA30022); estrada Mauá, igapó, col. G.T. Prance et al. 11730, 5-IV-1971 (INPA30042); rio Purus between lago Quati \& lago Arimã near rio Jacaré, col. G.T. Prance et al. 13418, 20-IV-1971 (INPA.31782); Fortaleza, Savana, rio Puciari tributary of rio Ituxi, $20 \mathrm{~km}$ above mouth, col. G.T. Prance et al. 13842, 1-VII-1971 (INPA32058), rio Curuquetê, vicinity of cachoeira São Bento, col. G.T. Prance et al. 14303, 14-VII-1971 (INPA32518); rio Purus, rio Curuquetê, vicinity of cachoeira São Bento, col. G.T. Prance et al. 14334, 15-VII-1971 (INPA32550); rio Cuieras just below mouth of rio Bracinho, col. G.T. Prance et al. 14937 , 27-IX-1971 (INPA33153); baixo rio Negro, Tapuruquara beside road to airport, col. G.T. Prance et al. 15290, 16-X-1971 (INPA33506); rio Solimões, path along bank between Leticia and EI Marco, col. G.T. Prance et al. 16833, 24-VII-1973, det. O. Yano (INPA40023); ManausItacoatiara road, $\mathrm{km}$ 69-70, col. G.T. Prance et al. (P17518) 5-IX-1973, det. D. G.iffin (INFA40714); Manaus. Reserva Ducke, km 26 Manaus-Itacoatiara road, col. C.C. Berg et al. P17605, 6.XI-1973, det. O. Yano (INPA40802, SP147608); Manaus, Reserva Florestal Ducke, km 26 da Manaus-Itacoatiara road, damp forest near stream, col. G.T. Prance et al. 18730, 
8-VIII-1973, det. O. Yano (INPA42053, SP147609); road Manaus-Caracaraí, km 125, co!. C.C. Berg et al. P19485, 12-XI-1973 (INPA42975); road Manaus-Porto Velho (BR-319) behind road camp, km 240, col. E. Lleras et al. P19596, P19597, 21-XI-1973, det. D. Griffin (INPA43080; 43082); Manaus-Porto Velho BR-319 behind road camp, km 240, col. E. Lleras et al. P19601, 22-XI-1973, det. D. Griffin (INPA40798); BR-319, Manaus-Porto Velho road, km 245, 3 km South of igapó Açu, col. G.T. Prance et al. 20457. 13-III-1974, det. O. Yano (INPA44435); Reserva Biológica Campina, estrada Manaus-Caracaraí, km 62, col. R. Lisboa 35, 13-XI-1974, det. D. Griffin (INPA48818); Manaus, Reserva Florestal Ducke, km 26 da Manaus-Itacoatiara, col. P. Lisboa 37, 9-VIII-1974, det. O. Yano (INPA49098, SP147612); Manaus, Reserva Campina, along the road Manaus-Caracaraí, km 60, col. D. Griffin, D.M. Vital \& O. Yano 6, 18, 8-VII-1974 (SP125226; SP125233); Manaus. Reserva Ducke, col. D. Griffin, D.M. Vital \& O. Yano 169, 195, 11-VII-1974 (SP125307; SP125322); Manaus, Tarumã Alta, sobre rochas, col. D. Griffin, D.M. Vital \& O. Yano, 279, 23-VII-1974 (SP125361); Manaus, Ponta Negra, col. D. Griffin, D.M. Vital \& O. Yano 284, 285 , 23-VII-1974 (SP125365; SP125366); Manaus, Reserva Ducke, igarapé Acará, col. D. Griffin, D.M. Vital \& O. Yano 339, 23-VII-1974 (SP125391); Manaus, ao longo da rodovia Manaus-Caracaraí, $\mathrm{km} \mathrm{130}$, dentro da caverna, col. D. Griffin, D.M. Vital \& O. Yano 441, 25-VII-1974, det. O. Yano (SP125426); Manaus, rodovia Manaus-Caracaraí, $\mathrm{km} \mathrm{60,} \mathrm{Reserva}$ Campina, col. D. Griffin, D.M. Vital \& O. Yano 564, 592A, 1-VIII-1974 (SP125464; SP125475); Manaus, rodovia Manaus-Caracaraí, km 115, rio Urubu, col. D. Griffin, D.M. Vital \& O. Yano 880, 13-VIII-1974 (SP125600); Manaus, rodovia Manaus-Caracaraí, km 60, Reserva Campina, col. D. Griffin, D.M. Vital \& O. Yano 943 , 963, 15-VIII-1974 (SP125623; SP125630); Manaus, rodovia Manaus-Caracaraí, km 60, Reserva Campina, col. D. Griffin, D.M. Vital \& O. Yano 991, 15-VIII-1974, det. P.A. Florschütz (SP125646); "Manaus, Reserva Egler, km 70 da estrada Manaus-Itacoatiara, col. R. Lisboa 254, 14-I-1977, det. O. Yano (INPA63765, SP147675);
Manaus, Reserva Ducke, km 26 da estrada Manaus-Itacoatiara, col. R. Lisboa 331, 336, 11-III-1976, det. R. Lisboa (INPA63842, SP147684; INPA63847, SP147686); col. R. Lisboa 344, 5-IV-1977, det. O. Yano (INPA70260, SP147717); Reserva Biológica de Campina, km 45 da estrada Manaus-Itacoatiara, col. R. Lisboa 356, 12-VIII-1977, det. O. Yano (INPA70272, SP147722, mixed Fissidens); col. R. Lisboa 359, 12-VIII-1977, det. O. Yano (INPA70275, SP147723); Manaus, igarapé Acará, Reserva Florestal Ducke, km 26 da estrada Manaus-Itacoatiara, col. P. Lisboa \& R. Lisboa 898, 913, 922, 937, 939, 6-1-1977, det. O. Yano (INPA62564, SP147657; INPA62584, SP147661; INPA62588, SP147662; INPA62603, SP147665; INPA62605, SP147666); Manaus, km 118 da estrada Manaus-Itacoatiara, col. P. Lisboa 998, 15-VI-1977, det. O. Yano (INPA66576, SP147699); rio Jaú, afluente do rio Negro, col. J. Ramos et al. 719, 7-V-1977, det. O. Yano INPA72672, SP147780); rio Jaú, sítio Fortaleza, col. J. Ramos et al. 723, 7-IV-1977, det. O. Yano (INPA73676, SP147781); cordilheira do Pico da Neblina, caminho para o rio 31 de Março à $1500 \mathrm{~m}$ alt., perto do Acampamento, col. W.A. Rodrigues 9831, 7-XI-1977, det. O. Yano (INPA71491, SP147805); Tarumã Grande, along rio Tarumã, co!. W.R. Buck 1784, 9-XI-1977, det. O. Yano (SP147785); cidade de Manaus, col. W.D. Reese 12682, 6-12-I-1978, det. O. Yano (INPA); rio Uatumã, $4 \mathrm{~km}$ da cachoeira, col. P. Lisboa et al. 1020, 1050, 1057, 20-II-1978, det. O. Yano (INPA75416; 75446; 75453); rio Uatumã, $4 \mathrm{~km}$ da cachoeira, col. P. Lisboa et al. 1081, 1083, 20-II-1978, det. R. Lisboa (INPA75475; 75477); rio Uatumã, lado direito, vegetação de campina, col. P. Ivo S. Braga et al. 3519, 27-II-1978, det. O. Yano (INPA75343, SP147726); estrada Manaus-Itacoatiara, km 52, Ramal SFI, que liga a ManausCaracarai, km 9, col. P. Lisboa et al. 1149, 1158, 9-IV-1978, det. O. Yano (INPA76382, SP147772; INPA76391, SP147773). Mato Grosso: Mun. de Aripuanã, Dardanelos, alto do Salto das Andorinhas, col. P. Lisboa et al. 262, 26-IX-1975, det. R. Lisboa (INPA52790, SP147625); Mun. de Aripuanã, col. C. Damião 767, 769, 14-II-1977, det. R. Lisboa (INPA66212, SP147690; INPA66214, SP147691); col. C. Da- 
mião, 18-II-1977, det. R. Lisboa (INPA66218, SP147693); col. C. Damião 781, 19-II-1977, det. R. Lisboa (INPA66226, SP147696); Aripuanã, col. O.P. Monteiro \& J. Leite 1101, 28-V-1976, det. O. Yano (INPA58934, SP147656). Pará: Breves, col. J.M. Pires et al. 4872, 7-30-VII-1956, det. O. Yano (IAN90370); Mun. de Óbidos, rio Jaramacaru, col. W.A. Egler 507, 7-VI-1957, det. O. Yano (MG23176, IAN39007); Pitoró, Região Igarapé, col. R.L. Fróes 34724, 26-IX-1958, det. O. Yano (IAN103543); rio Trombetas, vicinity of cachoeira Porteira, col. G.T. Prance et al. 22188, 22190, 20-V-1974, det. O. Yano (INPA46124; 46126); serra do Cachimbo, cachoeira Cucuá, base da cachoeira com spray constante, alta umidade, $340 \mathrm{~m}$, col. E. Lleras \& Kirkbride 1144, 15-II-1977, det. O. Yano (INPA64195). Território do Amapá: rio Jari, cachoeira das Guaribas $0^{\circ} 24^{\prime} \mathrm{N}, 53^{\circ} 7^{\prime} \mathrm{W}$, elev. ca $110 \mathrm{~m}$, col. W.A. Egler \& H.S. Irwin 46455 , 16-VIII-1961, (NY, SP148776). Território de Rondônia: Guajará-Mirim, km 20 da estrada Saldanha, col. E. Lleras et al. 1101, 1102, 12-1-1977, det. O. Yano (INPA63400; 63401); rio Pacaás Novos, sobre primeira Cachuera, col. W.D. Reese 13450, 19-III-1978, det. O. Yano (SP147759); col. W.D. Reese 13510, 20-III-1978, det. O. Yano (INPA). Território de Roraima: Vicinity of Auaris, $64^{\circ} 25^{\prime} \mathrm{W}, 4^{\circ} 6^{\prime} \mathrm{N}, 800 \mathrm{~m}$ alt., col. G.T. Prance et al. 21336, 23-VII-1974 (INPA45312); acampamento do 6..$^{\circ}$ BEC-Jundiá, km 328 on Manaus Caracaraí road, from junction at Manaus-Itacoatiara road, col. W.R. Buck 1846, 17-XI-1977, det. O. Yano (SP147791); Along Manaus-Caracarai road at $\mathrm{km} \mathrm{343,6} \mathrm{km}$ S of rio Branquinho, col. W.R. Buck 1878, 18-XI-1977, det. O. Yano (SP147794); Boca da Mata, $216 \mathrm{~km} \mathrm{~N}$ of Boa Vista, on Boa Vista-Caracas road, col. W.R. Buck 2012, 30-XI-1977, det. O. Yano (SP147803). Ocorre com muita freqüência na região amazônica o que não acontece em outras regiões brasileiras. Cresce em troncos de árvores vivas, em decomposição, na base, raízes e tronco de palmeiras, sobre casca de árvore e sobre pedras.

Octoblepharum rhaphidostegium C. Muell. ex Broth. Mato Grosso: rio Aripuanã, margin of new airstrip of Humboldt Center $59^{\circ} 21^{\prime} \mathrm{N}$, $10^{\circ} 12^{\prime} \mathrm{S}$, col. G. T. Prance et al. 18367,
10-X-1973, det. O. Yano (INPA41669); Mun. de Aripuanã, Estação Humboldt, col. C. Damião 2475, 26-III-1977, det. R. Lisboa (INPA69501, SP147712). Território de Rondônia: serra dos Murales, $14 \mathrm{~km}$ NNW of junction of rio Madeira and Abuná, col. G.T. Prance et al. 6024, 14-VII-1968, det. O. Yano (INPA22780); rio Pacaás Novos, sobre primeira Cachuera, col. W.D. Reese 13333, 15-22-III-1978, det. O. Yano (INPA). Território de Roraima: serra Parima, col. Bruce Nelson 21337, 21343, 28-VII-1974, det. O. Yano (SP125525, 125530). O. rhaphidos. tegium é semelhante a $O$. albidum, mas os filídios mais largos e delicados. Cresce nas rochas, nos cipós e troncos de árvores vivas. Está sendo a $1 .^{\mathrm{a}}$ citação para a região amazônica.

Octoblepharum stramineum Mitt. Acre: Cruzeiro do Sul, próximo ao aeroporto Novo, Projeto Radam/Brasil, col. O.P. Monteiro \& C. Damião 386, 14-1I-1976, det. O. Yano (INPA56236, SP147649). Amazonas: Basin of rio Negro, rio Uneiuxi, $100-200 \mathrm{~km}$, above mouth, black water flooded savana forest on sand, col. G.T. Prance et al. 15477, 21-X-1971, det. O. Yano (INPA33694); Manaus, Reserva Experimental do INPA, Manaus-Caracarai km 61. col. G.T. Prance et al. 18705, 3-VIII-1973 (INPA42018); rio Cuieras just below mouth of rio Brancinho, col. G.T. Prance et al. 14868, 25-IX-1971, det. O. Yano (INPA33084); Reserva Experimental do INPA, Manaus-Caracaraí road, km 61, col. G.T. Prance et al. 18711, 3-VIII-1973, det. R. Lisboa (INPA42024); rio Cuieras, $2 \mathrm{~km}$ below mouth of rio Brancinho, col. G.T. Prance et al. 17759, 11-IX-1973, det. O. Yano (INPA41063); Manaus, Reserva Campina, along the road Manaus-Caracaraí, $\mathrm{km} \mathrm{61,} \mathrm{col.} \mathrm{D.}$ Griffin, D.M. Vital \& O. Yano 32, 8-VII-1974, det. P.A. Florschütz (SP125238); col. D. Gr.ffin, D.M. Vital \& O. Yano 36, 37, 8-VII-1974 det. O. Yano (SP125241; SP125242, mixed Micropterygium); col. D. Griffin, D.M. Vital \& O. Yano 536, 1-VIII-1974, det. O. Yano (SP125451); Manaus, Reserva Biológica de Campina, BR-174, km 62, col. R. Lisboa 127, 11-IV-1975, det. D. Griffin (INPA49777); Reserva Biológica de Campina on Manaus-Caracaraí road, col. W.R. Buck 1763, 6-XI-1977, det. O. Yano (INPA); rio 
Uatumã, lado esquerdo do rio, vegetação de campina, col. P. Ivo S. Braga et al. 3497, 3512, 24-II-1978, det. R. Lisboa (INPA75321; INPA 75335, SP147725). Esta espécie conhecida da região amazônica por Mitten (1869) e Lisboa (1976). Fácil a identificação pela sua coloração paleáceae-brilhante, geralmente crescendo nos troncos de árvore viva, nas areias brancas próximo à troncos de árvores da campinarana, e as vezes nos ramos de árvores.

\section{Comentários}

A flora amazônica é tão vasta que foram realizadas apenas coletas localizadas, faltando cobrir uma boa área do Estado do Amazonas, Pará, Mato Grosso e Território do Amapá. Os Estados do Maranhão e Goiás não apresentam representantes de Leucobryaceae. Isto devido, realmente, à falta de coleta nestas áreas, porque estão dando prioridade às áreas mais atingidas pela devastação das matas.

Leucobryceae na regiảo amazônica brasileira está representada por três gêneros: Leucobryum Hampe com 6 espécies, Octoblepharum Hedw. com 10 espécies e uma variedade, e Ochrobryum com 3 espécies.

Dentre as 473 exsicatas examinadas, 121 são de Leucobryum martianum, 100 de Octoblepharum pulvinatum e 96 de Octoblepharum albidum. Isto confirma as nossas observações no campo, porque Leucobryum martianum ocorre com maior frequêencia que as outras duas espécies.

Ochrobryum parvulum nåo foi reencontrada na região como consta da literatura.

Octobiepharum stramineum é muito comum na região de "Campina ou Campinarana", principalmente na base dos troncos de árvores.

Das 20 espécies encontradas, 9 constituem a primeira citação para a região em estudo: Leucobryum albidum, L. bowringii, L. giganteum, L. juniperoideum, Octoblepharum albidum var. violascens, $O$. africanum, $O$. erectifolium, O. rhaphidostegium, Ochrobryum obtusifolium.

\section{Agradecimentos}

O autor deseja aqui expressar a mais profunda gratidão ao Conselho Nacional de Desenvolvimento Científico e Tecnológico $(\mathrm{CNPq})$, à Fundação de Amparo à Pesquisa do Estado de São Paulo (FAPESP), ao Instituto Nacional de Pesquisas da Amazônia (INPA) e Instituto de Botânica de São Paulo, o suporte financeiro oferecido para a realização deste trabalho. Ao Museu Paraense Emílio Goeldi (MG) e ao Instituto de Pesquisa e Experimentação Agropecuária do Norte, Belém (IAN), pela facilidade no uso dos Herbários e empréstimo do material.

\section{SUMMARY}

Nineteen species and one variety of Leucobryaceae are reported from the Brazilian Amazonian region, distributed in three genera: Leucobryum Hampe with six species, Octoblepharum Hedw. with ten species and one variety and Ochrobryum Mitt. with three species.

The following species are reported for the first time for the region: Leucobryum albidum ( $P$. Beauv.) Lindb., L. bowringii Mitt., L. giganteum C. Muell., L. juniperoideum (Brid.) C. Muell., Octoblepharum albidum var. violascens C. Muell., O. africanum (Broth.) Card., O. erectifolium Mitt. ex Williams, $\mathbf{O}$. rhaphidostegium C. Muell. ex Broth. and Ochrobryum obtusifolium (C. Muell.) Mitt. Leucobryum martianum (Hornsch.) Hampe, Octoblepharum pulvinatum (Dozy et Molk.) Mitt. and O. albidum Hedw. are the commonest species.

\section{REFERENNCIAS BIBLIOGRÁFICAS}

BESCHERELLE, Émile

1897 - Révision du genre Ochrobryum. J. Bot., 11: 138-153, fig. 1-7.

DUCKE, A. \& BLACK, G.A.

1954 - Notas sobre a fitogeografia da Amazônia Brasileira. Bolm. Téc. Inst. Agron. Norte, 29: 3-62, 1 map.

FLORSCHÜTZ, Peter A.

1964 - The mosses of Suriname. Flora of Suriname. Leiden. vol. 6: i-xxvii +1-271 p., fig. 1-107.

GRIFFIN, Dana

1979 - Guia preliminar para as briófitas freqüentes em Manaus e adjacências. Acta Amazonica, 9 (3): 1-67, fig. 1-536. 
LISBOA, Regina C.L.

1976 - Estudos sobre a vegetação das campinas amazônicas. 5. Brioecologia de uma campina amazônica. Acta Amazonica, 6 (2): 171-191, map. 1-6, tab. 1-10, 1 fot., graf. 1-2.

LISBOA, Regina C.L. \& LISBOA, Pedro L.B.

1978 - Contribuição ao conhecimento da flora do Aripuanã (Mato Grosso) II. Musci. Acta Amazonica, 8 (2): 143-148.

MITTEN, William

1869 - Musci austro-americani. Enumeratio muscorum omnium austro-americanorum hucusque cognitorum. J. Linn. Soc. Bot., 12: 1-659.

PRANCE, Gillean T.

1971 - An index of plant collectors in Brazilian Amazonia. Acta Amazonica, 1 (1): 25-64.

REESE, William D.

1979 - Calymperaceae (Musci) from Western Amazonia: Brazil and Bolivia. Bryologist, 82 (4): 559-563.
REESE, William D. \& GRIFFIN, Dana

1976 - Syrrhopoden annotinus (Musci, Calymperaceae), a new species from Amazonas, Brazil. Bryologist, 79 (4): 518-520, fig 1-10.

1977 - Noteworthy Calymperaceae from Brazil and Venezuela. Acta Amazonica, 7 (2): 179-184.

SPRUCE, Richard

1867 - Catalogus muscorum fere omnium quos in Terris Amazonicis et Andinis, per annos 1849-1860. Londono, $\mathrm{i}+1-22$.

YANO, Olga

1975 - Leucobryaceae (Musci) do Estado de São Paulo. São Paulo: p. i-iv $+1-176$ (Tese de Mestrado em farmacologia, Area de Produtos Naturais, Escola Paulista de Medicina).

(Aceito para publicação em 25/01/82) 